\title{
Pegada hídrica da alimentação de adolescentes do Brasil: relações com o consumo de
}

\section{fast food e o local de moradia}

\author{
Water footprint of the diet of adolescents in Brazil: relationships with fast food consumption and \\ place of residence
}

Huella hídrica de la dieta de los adolescentes en Brasil: relaciones con el consumo de comida rápida y lugar de residencia

Recebido: 14/09/2021 | Revisado: 25/09/2021 | Aceito: 27/09/2021 | Publicado: 29/09/2021

Diôgo Vale
ORCID: https://orcid.org/0000-0003-2636-4956
Instituto Federal de Educação, Ciência e Tecnologia do Rio Grande do Norte, Brasil
E-mail: diogo.vale@escolar.ifrn.com.br
Natalie Marinho Dantas
ORCID: https://orcid.org/0000-0003-1763-3472
Universidade de São Paulo, Brasil
E-mail: natiedantas@gmail.com
Camila Valdejane Silva de Souza
ORCID: https://orcid.org/0000-0001-7146-3406
Universidade Federal do Rio Grande do Norte, Brasil
E-mail: camila.valdejane@gmail.com
Maria Hatjiathanassiadou
ORCID: https://orcid.org/0000-0001-9367-1230
E-mail: mariahatji@ Norte, Brasil.com
Universidade Federal do Rio Grande do
Larissa Mont'Alverne Jucá Seabra
ORCID: https://orcid.org/0000-0002-1878-4283
Universidade Federal do Rio Grande do Norte, Brasil
E-mail: larissa.seabra@ufrn.br

\section{Resumo}

O objetivo deste estudo foi estimar as pegadas hídricas da alimentação de adolescentes escolares do Brasil e as associações com contexto territorial e de rotina alimentar. Trata-se de um estudo ecológico a partir dos microdados da amostra 1 da Pesquisa Nacional de Saúde do Escolar 2015, com as quais foram estimadas as médias das pegadas hídricas medias $(\mathrm{PH})$ da alimentação dos adolescentes por categoria de análise: unidade da federação brasileira, macrorregião geográfica e por número de dias na semana frequentado restaurantes fast food. Estatísticas descritivas e espaciais foram empregadas para compreensão da distribuição dessas médias nas unidades de análise territoriais e na frequência em fast food. No Brasil, a PH média da alimentação dos adolescentes foi 2925,9 litros $/ \mathrm{kg}$. A distribuição das PH apresentou forte correlação espacial (Índice de Moran Local=0,773), com destaque para formação do cluster do tipo alto-alto (Rio Grande do Sul, Santa Catarina, Paraná, São Paulo, Rio de Janeiro, Espírito Santo, Minas Gerais e Mato Grosso do Sul). Além disso, as PH médias da alimentação desse grupo aumentaram proporcionalmente ao aumento do número de dias que o adolescente costumava comer em fast food. Percebe-se uma relação direta entre o maior impacto ambiental da alimentação de adolescentes, territórios de moradia mais urbanizados e a maior frequência em restaurantes fast food. Avaliar essas associações é importante para compressão das questões de saúde pública e nutrição na perspectiva da sustentabilidade. Assim, podem ser desenvolvidas estratégias para redução do impacto ambiental e melhoria da alimentação desse grupo etário.

Palavras-chave: Adolescente; Indicadores de desenvolvimento sustentável; Alimentação; Impacto ambiental.

\begin{abstract}
The aim of this study was to estimate the water footprints of the diet of teenage schoolchildren in Brazil and the associations with territorial context and food routine. This is an ecological study based on microdata from sample 1 of the 2015 National Adolescent Health Survey, with which the average water footprint (WF) of adolescents' nutrition was estimated by category of analysis: Brazilian federation unit, geographic macro-region and by number of days a week attended fast food restaurants. Descriptive and spatial statistics were used to understand the distribution of these means in territorial analysis units and frequency in fast food. In Brazil, the average WF of the adolescents' diet was 2925.9 liters $/ \mathrm{kg}$. The distribution of WF showed a strong spatial correlation (Moran Local Index=0.773), with emphasis on the formation of the high-high cluster (Rio Grande do Sul, Santa Catarina, Paraná, São Paulo, Rio de
\end{abstract}


Janeiro, Espírito Santo, Minas Gerais and Mato Grosso do Sul). In addition, the mean WF of this group's diet increased proportionally to the increase in the number of days that the adolescent used to eat at fast food. A direct relationship can be seen between the greater environmental impact of eating adolescents, more urbanized living areas and greater attendance at fast food restaurants. Assessing these associations is important for compressing public health and nutrition issues from the perspective of sustainability. Thus, strategies can be developed to reduce the environmental impact and improve the diet of this age group.

Keywords: Adolescent; Sustainable development indicators; Diet; Environmental Impact.

\section{Resumen}

El objetivo de este estudio fue estimar las huellas hídricas de la dieta de los escolares adolescentes en Brasil y las asociaciones con el contexto territorial y la rutina alimentaria. Se trata de un estudio ecológico basado en microdatos de la muestra 1 de la Encuesta Nacional de Salud del Adolescente 2015, con el cual se estimó la huella hídrica (HH) promedio de la nutrición de los adolescentes por categoría de análisis: unidad federativa brasileña, macrorregión geográfica y por número de días a la semana asistía a restaurantes de comida rápida. Se utilizó estadística descriptiva y espacial para comprender la distribución de estas medias en las unidades de análisis territorial y frecuencia en comida rápida. En Brasil, el HH promedio de la dieta de los adolescentes fue de 2925,9 litros / kg. La distribución de PH mostró una fuerte correlación espacial (Índice Local de Moran = 0,773), con énfasis en la formación del clúster alto-alto (Rio Grande do Sul, Santa Catarina, Paraná, São Paulo, Rio de Janeiro, Espírito Santo, Minas Gerais y Mato Grosso do Sul). Además, el HH medio de la dieta de este grupo aumentó proporcionalmente al aumento en el número de días que el adolescente solía comer en comida rápida. Existe una relación directa entre el mayor impacto ambiental de la alimentación de adolescentes, las áreas de vida más urbanizadas y la mayor frecuencia de restaurantes de comida rápida. La evaluación de estas asociaciones es importante para comprimir los problemas de salud pública y nutrición desde la perspectiva de la sostenibilidad. Así, se pueden desarrollar estrategias para reducir el impacto ambiental y mejorar la dieta de este grupo de edad.

Palabras clave: Adolescente; Indicadores de desarrollo sostenible; Dieta; Impacto medioambiental.

\section{Introdução}

O atual padrão alimentar causa impactos negativos não apenas na saúde humana, mas também no meio-ambiente. Esse é resultado da transição alimentar global que se caracteriza pela substituição de uma alimentação tradicional por alimentos com alto teor de gorduras e açúcares refinados, óleos, produtos cárneos e aditivos sintéticos. Ressalta-se que caso não ocorra modificação dessa tendência, a rede de aspectos relacionadas à alimentação (produção, distribuição, consumo e resíduos) irá se consolidar em 2050 com um dos principais fatores responsáveis pela degradação ambiental (Tilman \& Clark, 2014; Aleksandrowicz et al., 2016; Swinburn et al., 2019).

Nesse contexto, o uso de água é um dos principais indicadores do efeito humano no planeta. Sabe-se que a produção de alimentos atualmente é considerada o maior consumidor desse bem natural em nível global. Estima-se, por exemplo, que a agricultura é responsável por $70 \%$ do uso de água doce, tendo esse valor mais do que duplicado entre os anos de 1961 e 2000. Discute-se ainda que a possibilidade para o uso de água doce já ultrapassou o seu limite planetário, o que aumenta as chances de gerar mudanças irreversíveis ao meio ambiente. Com base nisso, e tendo em vista o aumento populacional, a alimentação como necessidade humana básica e a transição alimentar global, é necessário repensar a utilização de recursos naturais, como a água, nos sistemas alimentares (Campbell et al., 2017; Gerten et al., 2015; Willet et al., 2019).

A superação desses desafios demanda ações coletivas para promoção de uma alimentação mais saudável e sustentável. Essas devem estar em consonância com as agendas de desenvolvimento mundial, como os Objetivos do Desenvolvimento Sustentável (United Nations, 2015). Nesse sentido da promoção de hábitos de vida, sabe-se da importância da ação precoce, principalmente junto a crianças e adolescentes, porque são um público em formação e com incidência social e política a médio e longo prazo. No âmbito da alimentação e do desenvolvimento sustentável, é urgente o fortalecimento de atividades com adolescentes pois esses constituem um público estratégico para esse tipo ação (Sheehan et al., 2017).

A adolescência é um período da vida no qual as necessidades nutricionais estão mais acentuadas e demandam, por isso, maior consumo energético e proteico. Além disso, são um grupo que se caracteriza pelo desenvolvimento de sua autonomia para escolhas alimentares e, em muitos casos, acabam optando mais por alimentos ultraprocessados (Vale, 2020a). 
No Brasil, por exemplo, resultados da avaliação de consumo alimentar de adolescentes apontaram um maior consumo de ultraprocessados, com destaque para os embutidos cárneos, em comparação aos adultos e idosos (IBGE, 2020). Todas essas características nutricionais e dietéticas relacionam-se ao maior impacto ambiental da alimentação quando avaliados indicadores como as pegadas ecológicas, hídrica e de carbono entre adolescentes (Han, Chai \& Liao, 2020).

Essas características corroboram os resultados das pesquisas que demonstraram padrões alimentares com alto consumo de alimentos de origem animal e ultraprocessados associados ao maior impacto ambiental negativo (Hallström, Carlsson-Kanyama \& Börjesson, 2015). Ao contrário disso, padrões culturais como a dieta mediterrânea, por exemplo, apresentaram menor impacto ambiental, destacando-se a necessidade de alterações na ingestão alimentar como necessárias para se preservar a saúde humana e ambiental (Grosso et al., 2020; Naja et al., 2020).

Com base na necessidade de discussão, avaliação e monitoramento desses aspectos, emergiu o conceito de Nutrição Sustentável o qual tem como base cinco dimensões (saúde, ambiente, economia, social e cultural). Para concretização dessa ideia são propostas ações, como a preferência por uma alimentação à base de plantas, com alimentos orgânicos, sazonais, minimamente processados, que respeite a cultura alimentar, sendo oriundo de um comércio justo e que tenha um uso sustentável dos recursos (Von Koerber, Bader \& Leitzmann, 2017).

Dentre os diversos fatores que podem estar associados à uma alimentação sustentável pode-se citar a frequência de consumo e o tamanho das porções dos alimentos de origem animal. Esses alimentos, em especial a carne vermelha, demandam muitos recursos naturais e ambientais para serem produzidos, emitindo valores consideráveis de GEE na atmosfera (19\% de todos os GEE emitidos), o maior uso de água para produção quando comparados com os outros alimentos e demandando grandes quantidades de terra, que estão associados diretamente com o desflorestamento e a perda da biodiversidade (Swinburn et al., 2019; Mekonnen \& Gerbens-Leenes, 2020; Seabra et al., 2021).

Além desses alimentos de origem animal, também deve-se considerar o impacto ambiental negativo de alimentos ultraprocessados, como embutidos, salgados de pacote e guloseimas. Na Austrália, o consumo de alimentos ultraprocessados contribui com mais de um terço de todos os impactos ambientais relacionados à dieta (sendo 35\% de uso de água; 39\% de uso de energia e 33\% de emissão de GEE) (Monteiro et al., 2019). É importante destacar também que as avaliações dos impactos ambientais dos alimentos ultraprocessados atualmente não consideram os processos industriais e a grande variedade de compostos utilizados, como os aditivos alimentares. Ao serem considerados esses itens, os indicadores de impacto ambiental certamente aumentarão ainda mais. Associado a isso, temos o fato de que os ultraprocessados usam extensas quantidades de embalagens, sendo uma importante fonte de produção de resíduos (Seferidi et al., 2020)

Nesse sentido, o uso de indicadores como a pegada hídrica - quantidade total de água utilizada direta ou indiretamente durante as fases do ciclo de vida do alimento estimada a partir da somatória da água de superfície ou subterrânea (água azul), com a água de chuva (água verde) e a água necessária para assimilar a carga de poluição do sistema de produção e consumo (água cinza) (Garzillo et al., 2019) - pode servir de base para reconhecimento do impacto ambiental da alimentação dos grupos populacionais. Com adolescentes, essas estimativas podem gerar ações educativas e políticas públicas que promovam o consumo de alimentos com menor impacto negativo no ambiente (Hatjiathanassiadou et al 2019; Vale, 2020b). Com isso, o objetivo deste estudo foi estimar as pegadas hídricas da alimentação de adolescentes escolares do Brasil e as associações com contexto territorial e de rotina alimentar.

\section{Metodologia}

Trata-se de um estudo ecológico cujas unidades de análise foram as macrorregiões geográficas e as unidades da federação brasileiras, e a frequência semanal em restaurantes fast food. As estimativas da média das pegadas hídricas (PH) da alimentação de cada adolescentes foi realizada a partir dos dados individuais disponíveis nos microdados da amostra 1 da 
Pesquisa Nacional de Saúde do Escolar 2015 (PeNSE 2015) - estudo transversal e populacional. Nessa terceira edição da PeNSE, foram coletados dados de 102.072 adolescentes do nono ano de escolas públicas e privadas de 27 unidades da federação brasileiras a partir de um desenho amostral complexo. Durante a coleta, os adolescentes responderam a um questionário auto aplicado em dispositivo digital (IBGE, 2016).

Esse estudo de base escolar foi realizado pelo Instituto Brasileiro de Geografia e Estatística (IBGE), Ministério da Saúde e Ministério da Educação do Brasil com aprovação da Comissão Nacional de Ética em Pesquisa (Conep), em 30 de março de 2015 (registro $\mathrm{n}^{\circ}$ 1.006.467). O presente trabalho atende às questões de ética em pesquisa dispostas na resolução $\mathrm{n}^{\circ}$ 510 de 07 de abril de 2016 do Conselho Nacional de Saúde (Brasil, 2016). Outras informações sobre a metodologia da PeNSE 2015 estão disponíveis para consulta na publicação do IBGE (IBGE, 2016).

\subsection{Construção das variáveis}

\subsubsection{Estimativa da Pegada Hídrica da alimentação}

Os cálculos da estimativa da pegada hídrica da alimentação - litros de água gasta por quilograma de alimento (litros/kg) - foram realizados a partir dos seguintes passos: (1) estimativa das pegadas hídricas médias para cada um dos sete marcadores de alimentação da PeNSE 2015 (frutas, legumes e verduras, feijões, ultraprocessados salgados, refrigerantes, guloseimas, salgados fritos) segundo banco de Garzillo et al (2019) ; (2) estimativa de per capitas médios de 2015 para cada um desses sete marcadores de alimentação para espaços urbanos e rurais de cada uma das cinco regiões brasileiras com base nos dados das Pesquisas de Orçamentos Familiares (POF) de 2009 (IBGE, 2010) e de 2018 (IBGE, 2019); (3) desenvolvimento de fatores de pegadas hídrica por marcador de alimento, dia, local de moradia e região geográfica; e (4) estimativa das pegadas hídrica da alimentação de cada adolescente resultante do produto entre as pegadas hídricas de cada marcador de alimentos e sua frequência de consumo semanal (Vale et al., 2021).

\subsubsection{Variáveis secundárias}

A população de adolescentes foi caracterizada quanto à idade, ao sexo (masculino, feminino) e área de moradia (urbana, rural). Além disso, foram utilizadas variáveis relativas às unidades territoriais brasileiras onde cada adolescente residia: macrorregião geográfica (Norte, Nordeste, Sudeste, Sul e Centro-Oeste) e unidade da federação (UF) (Rondônia, Acre, Amazonas, Roraima, Pará, Amapá, Tocantins, Maranhão, Piauí, Ceará, Rio Grande do Norte, Paraíba, Pernambuco, Alagoas, Sergipe, Bahia, Minas Gerais, Espírito Santo, Rio de Janeiro, São Paulo, Paraná, Santa Catarina, Rio Grande do Sul, Mato Grosso do Sul, Mato Grosso, Goiás, Distrito Federal).

O consumo semanal de alimentos em restaurantes fast food pelos adolescentes foi estimado com base na variável coletada a partir da seguinte pergunta da PeNSE: "nos últimos 7 dias, em quantos dias você comeu em restaurantes fast food, tais como lanchonetes, barracas de cachorro-quente, pizzaria etc?". O resultado variou de nunca (0 dias/semana) até todos os dias (7 dias/semana).

\subsection{Análise de dados}

A população de adolescentes escolares foi caracterizada com base em análise de frequências das variáveis idade, sexo e localização do domicílio. Para análise descritiva dos dados utilizou-se o software Statistical Package for the Social Science (SPSS) a partir do qual foi utilizado o efeito do desenho amostral complexo para estimativa das médias e respectivos intervalos de confiança (95\%) das PH da alimentação de adolescentes nas categorias geográficas - Brasil, macrorregião e cada uma das 27 UF - e de rotina alimentar - frequência de consumo de alimentos em fast food. 
Técnicas de análise espacial de dados de área a partir das malhas digitais brasileiras por UF foram empregadas utilizando software de Sistema de Informação Geográfica (SIG). Em todas as análises as PH da alimentação foram incluídas como variáveis de natureza contínua. A análise espacial foi realizada pelo software GeoDa 0.9.9.10 (Spatial Analysis Laboratory, University of Illinois, Urbana-Champaign, Estados Unidos). A distribuição das médias de PH de adolescentes no território brasileiro foi realizada a partir da construção de um cartograma de polígonos (27 UF) dividido em cinco faixas observadas no natural break map.

Para a identificação da autocorrelação espacial estatisticamente significativa $(\mathrm{p}<0,05)$ foi empregada uma análise univariada do tipo Local Indicators of Spatial Association. Essa técnica possibilita uma avaliação da autocorrelação espacial da variável principal (médias das PH da alimentação de adolescentes). Essa análise gera o Índice de Moral Local (IML) e os mapas de autocorrelação espacial do tipo Moran map. Os valores de correlação gerados pelo IML podem ser avaliados como positivos ou negativos, e como fracos $(<0,3)$, moderados $(0,3-0,7)$ ou fortes $(>0,7)$, como utilizado na avaliação da correlação de Pearson (Brasil, 2007a).

A interpretação dos aglomerados espaciais (clusters) gerados no Moran map pode ser de cinco tipos com as seguintes interpretações: não significativo (territórios que não entraram na formação de clusters, devido suas diferenças não terem sido significativas); alto-alto (regiões formadas por UF com altas médias da variável, circundada por regiões de altas médias); baixo-baixo (regiões formadas por UF com baixas médias da variável, circundada por regiões de baixas médias), alto-baixo (regiões formadas por UF com altas médias da variável, circundada por regiões de baixas médias), e baixo-alto (regiões formadas por UF com baixas médias da variável, circundada por regiões de altas médias) (Brasil, 2007a).

\section{Resultados e Discussão}

Entre a população de adolescentes estudantes avaliados a maioria tinha 14 anos (50,6\%), 15 anos (20,4\%) e 13 anos (16,4\%). Dentre todos, $51,7 \%$ eram do sexo feminino e $48,3 \%$, do sexo masculino; $35,6 \%$, da região Nordeste; $23,5 \%$, da Norte; $17,4 \%$, da Sudeste; $13,9 \%$, da região Centro-Oeste; e 9,7\%, da Sul; 91,9\% moravam em áreas urbanas e 8,1\%, em áreas rurais.

\subsection{Distribuição Espacial da Pegada Hídrica de Adolescentes}

No Brasil, a PH média da alimentação dos adolescentes avaliados foi 2925,9 litros $/ \mathrm{kg}$. Observou-se que os menores valores de PH da alimentação foram encontrados em adolescentes moradores de unidades da federação da região Norte: Amazonas (1777,9 litros/kg) e Pará (1803,4 litros/kg). E as maiores PH foram identificadas em adolescentes da região Sul: Rio Grande do Sul (3690,1 litros/kg) e Paraná (3701,5 litros/kg) (Tabela 1). 
Tabela 1. Pegada hídrica média da alimentação de adolescentes do Brasil, por Unidades da Federação, Brasil, 2015.

\begin{tabular}{|c|c|c|c|}
\hline \multirow[t]{3}{*}{ Unidade da Federação } & \multicolumn{3}{|c|}{ Pegada hídrica (litros/kg) } \\
\hline & \multirow[t]{2}{*}{ Média } & \multicolumn{2}{|c|}{ Intervalo de Confiança 95\% } \\
\hline & & Inferior & Superior \\
\hline Brasil & 2925,9 & 2625,8 & 3226,1 \\
\hline Região Norte & 1854,2 & 1731,3 & 1977,1 \\
\hline Rondônia & 1966,3 & 1894,9 & 2037,7 \\
\hline Acre & 1824,9 & 1517,5 & 2132,2 \\
\hline Amazonas & 1777,9 & 1461,7 & 2094,2 \\
\hline Roraima & 1963,3 & 1576,5 & 2350,1 \\
\hline Pará & 1803,4 & 1697,6 & 1909,2 \\
\hline Amapá & 1877,1 & 1744,0 & 2010,2 \\
\hline Tocantins & 2172,6 & 2141,1 & 2204,1 \\
\hline Região Nordeste & 2217,1 & 2117,5 & 2316,8 \\
\hline Maranhão & 1940,3 & 1828,6 & 2051,9 \\
\hline Piauí & 2100,9 & 1933,3 & 2268,6 \\
\hline Ceará & 2191,7 & 2068,3 & 2315,1 \\
\hline Rio Grande do Norte & 2208,1 & 2158,0 & 2258,2 \\
\hline Paraíba & 2354,6 & 2354,2 & 2355,0 \\
\hline Pernambuco & 2391,0 & 2348,1 & 2434,0 \\
\hline Alagoas & 2338,6 & 2278,8 & 2398,3 \\
\hline Sergipe & 2512,3 & 2476,9 & 2547,6 \\
\hline Bahia & 2198,5 & 2185,4 & 2211,6 \\
\hline Região Sudeste & 3419,2 & 3335,3 & 3503,1 \\
\hline Minas Gerais & 3365,5 & 3354,2 & 3376,8 \\
\hline Espírito Santo & 3067,7 & 3065,9 & 3069,5 \\
\hline Rio de Janeiro & 3346,2 & 3286,2 & 3406,2 \\
\hline São Paulo & 3497,6 & 3474,9 & 3520,2 \\
\hline Região Sul & 3640,3 & 3536,6 & 3744,0 \\
\hline Paraná & 3701,5 & 3683,5 & 3719,5 \\
\hline Santa Catarina & 3477,6 & 3424,8 & 3530,5 \\
\hline Rio Grande do Sul & 3690,1 & 3561,4 & 3818,9 \\
\hline Região Centro-Oeste & 2933,7 & 2830,7 & 3036,7 \\
\hline Mato Grosso do Sul & 2744,2 & 2560,8 & 2927,6 \\
\hline Mato Grosso & 2887,7 & 2800,3 & 2975,0 \\
\hline Goiás & 3041,8 & 3024,4 & 3059,3 \\
\hline Distrito Federal & 2908,7 & 2908,7 & 2908,7 \\
\hline
\end{tabular}

Fonte: Autores.

Um estudo com adolescentes libaneses demonstrou que a PH do consumo alimentar aumentou ao longo dos anos,

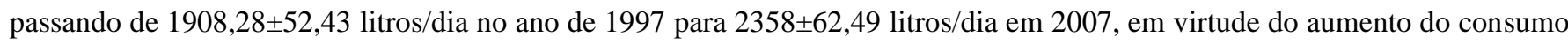
de pães e cereais, aves, peixes, salgadinhos e bebidas açucaradas. No geral, foram verificados valores de PH superiores aos valores estimados para a região Norte e inferiores aos observados entre os adolescentes brasileiros que residiam nas unidades da federação do eixo Centro-Sul do Brasil (Naja et al., 2020).

Verificou-se a formação de padrões espaciais das médias de PH de adolescentes nas unidades da federação brasileiras. Moradores do território Centro-Sul do Brasil apresentam maiores PH. Adolescentes de alguns estados do Nordeste (Rio 
Grande do Norte, Paraíba, Pernambuco, Alagoas e Sergipe) apresentam uma PH intermediária. Já adolescentes que viviam em estados da região Norte e no Maranhão (região Nordeste), apresentaram menores PH (figura 1a).

A aplicação de estatística espacial demonstrou forte correlação espacial (IML=0,773) com formação de um cluster do tipo alto-alto (Rio Grande do Sul, Santa Catarina, Paraná, São Paulo, Rio de Janeiro, Espírito Santo, Minas Gerais e Mato Grosso do Sul), e outro do tipo baixo-baixo (Maranhão, Pará, Roraima, Amapá). Esses clusters foram estatisticamente significativas (Figura 1b).

Figura 1. Distribuição espacial das médias de pegada hídrica (Natural break map) e dos clusters significativos dessas médias (Moran Map) da alimentação de adolescentes estudantes, por Unidades da Federação, Brasil, 2015.

a) Natural break map
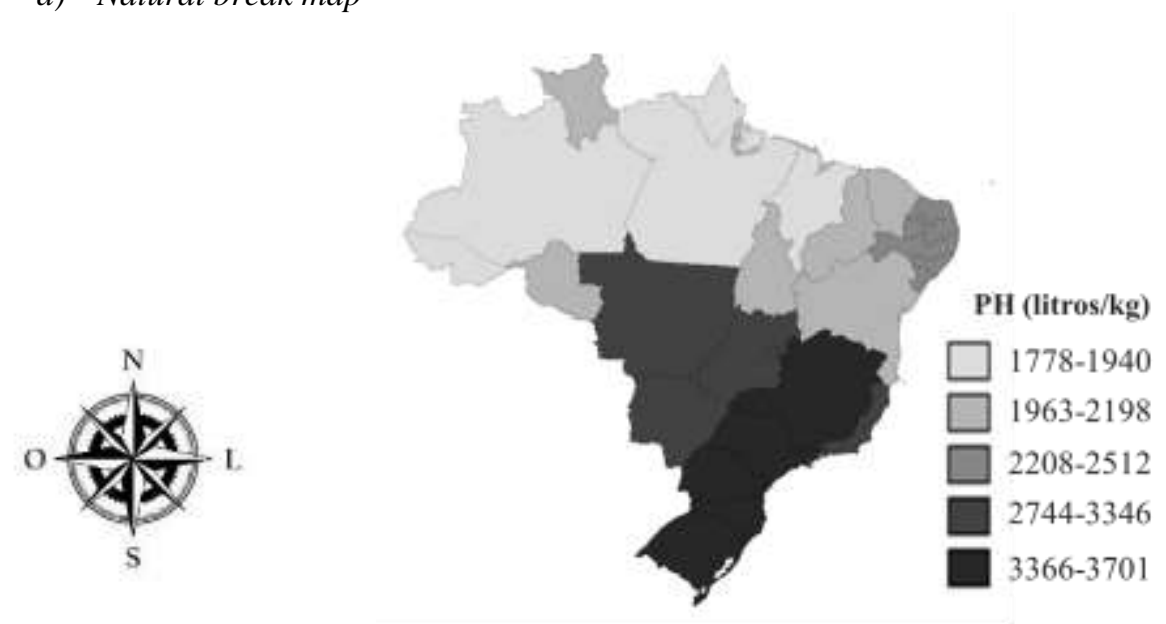

b) Moran map
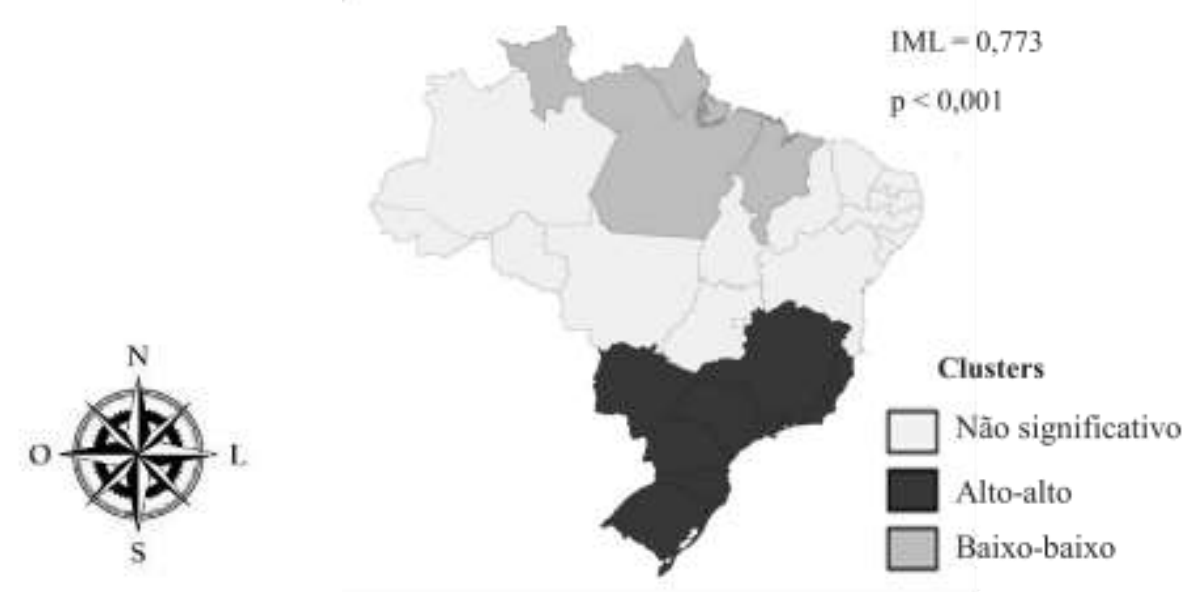

Fonte: Autores.

Analisar a distribuição espacial das estimativas de PH de marcadores do consumo alimentar no território brasileiro é imprescindível para compreensão dos seus impactos ambientais e dos fatores que influenciam as práticas alimentares de adolescentes. As regiões Sul e Sudeste brasileiras, que apresentaram maiores estimativas de PH de marcadores do consumo alimentar, concentram também as maiores taxas de urbanização e maior poder de compra com base no rendimento per capita. 
Enquanto as menores PH, foram identificadas na região Norte do país, que apresenta menor rede urbana (IBGE, 2021a; IBGE, 2021b). Uma pesquisa na Índia apontou PH maior em dietas de áreas urbanas, quando comparadas às áreas rurais que em geral apresentam uma alimentação com características tradicionais no país (Haris et al., 2017).

Sabe-se que territórios mais urbanizados e desenvolvidos favorecem a disponibilidade de produtos alimentícios ultraprocessados (OPAS, 2015). O Guia Alimentar para a População Brasileira ressalta que o consumo desses ultraprocessados impacta negativamente o meio ambiente e a sustentabilidade. Dentre os diversos impactos oriundos da produção desses alimentos, tem-se o grande volume de água utilizado (Brasil, 2014). Um estudo constatou que um em cada quatro adolescentes brasileiros referiram consumir diariamente ultraprocessados (Costa et al., 2018). Além disso, o maior consumo e disponibilidade desses alimentos em domicílios brasileiros foi identificado em unidades federativas das regiões Sul, Sudeste e Centro-Oeste brasileiras (Vale et al., 2019).

A urbanização tem relação direta com o meio ambiente. Além do impacto ambiental proporcionado pelas práticas alimentares nesses territórios com maiores taxas de urbanização, soma-se outras ações antrópicas observadas nesses espaços, como as emissões provenientes de veículos automotores e de indústrias, o consumo de energia, questões de saneamento ambiental, poluição hídrica e atmosférica urbanas, dentre outros aspectos que comprometem a sustentabilidade ambiental (Jatobá, 2011).

\subsubsection{Pegada Hídrica de Adolescentes de acordo com a frequência de consumo em restaurantes fast food}

Ao analisar a PH de adolescentes segundo o número de dias na semana que tinham frequentado restaurantes fast food, foi verificado um aumento progressivo desse indicador proporcional ao número de dias que o adolescente costumava comer nesses tipos de restaurante. Verificaram-se acréscimos de mais de mil litros de água por quilograma na alimentação de adolescentes que comiam todos os dias em restaurantes fast food, quando comparado àqueles que não haviam comido nesses estabelecimentos. Esse aumento de PH ocorreu entre os adolescentes de todas as grandes regiões do Brasil (Figura 2). 
Figura 2. Pegada hídrica $(\mathrm{L} / \mathrm{Kg})$ e frequência de consumo em restaurantes fast-food na última semana (dias/semana) entre adolescentes brasileiros por região. Brasil, 2015.
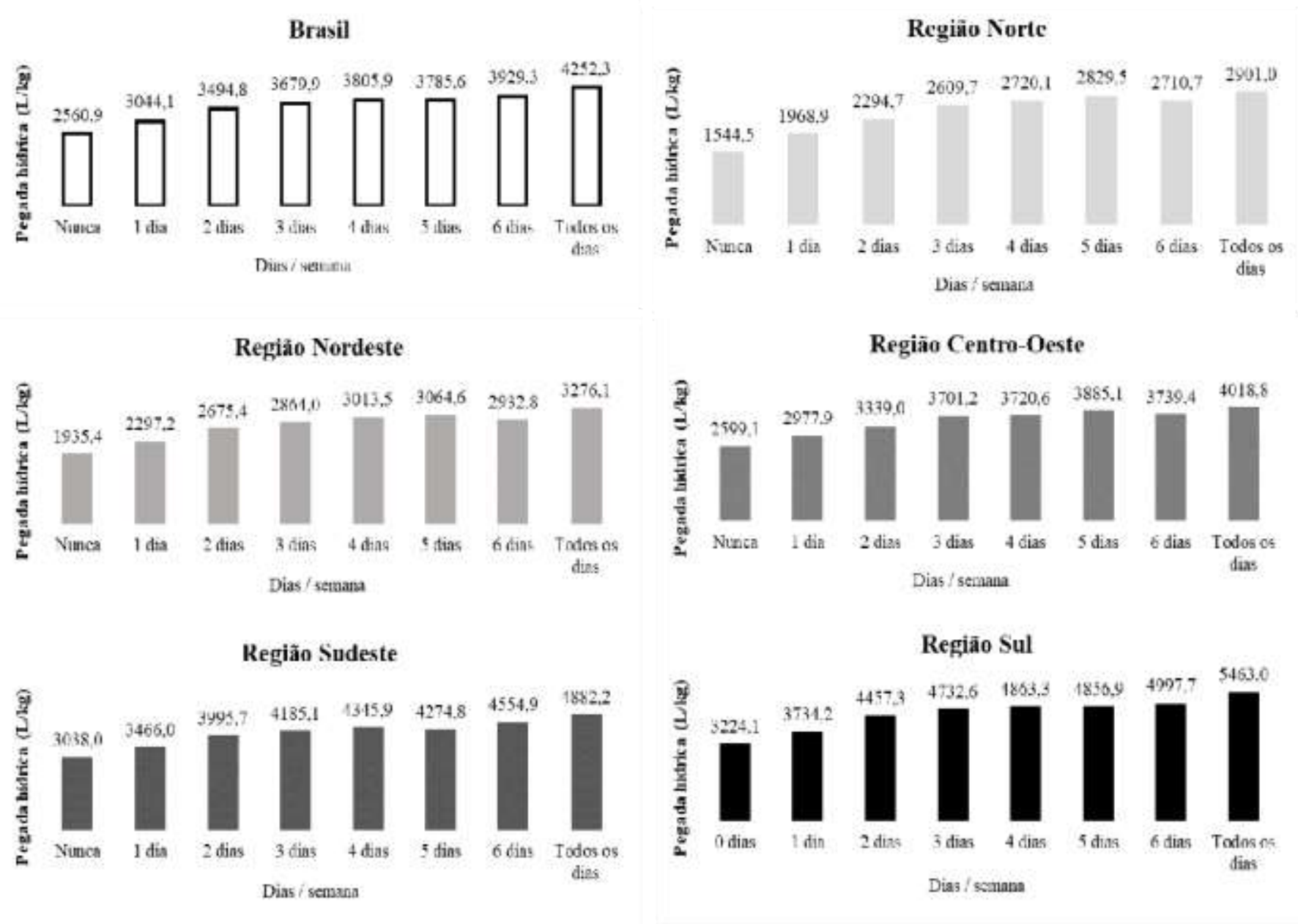

Fonte: Autores.

Realizar refeições em restaurantes fast food é um marcador para pior consumo alimentar pela baixa qualidade nutricional dos alimentos ofertados nesses espaços frequentemente utilizados para o consumo de alimentos fora do lar (Bezerra et al., 2017). No Brasil, o consumo de alimentos ultraprocessados é maior entre indivíduos que consomem refeições fora do lar, em comparação com o consumo de refeições feitas em casa. Por isso, a importância de ações e estratégias voltadas para a promoção de ambientes que ofereçam opções de alimentos mais saudáveis, menos processados e mais acessíveis, e que desestimulem o consumo de alimentos ultraprocessados fora de casa (Andrade et al., 2020). Sabe-se que fazer refeições nesses espaços piora a qualidade da dieta e aumenta o impacto ambiental, por isso o Guia alimentar para população brasileira orienta procurar locais que fornecem refeições "feitas na fora" quando precisar realizar refeições fora de casa (Brasil, 2014).

Esses espaços de comercialização de alimentos fast food além de repercutirem no maior impacto ambiental da alimentação, afetam também em outras dimensões da nutrição sustentável, como a cultura, a sociedade e a saúde. O consumo de alimentos em redes fast food, por exemplo, impacta de forma direta a cultura alimentar de uma sociedade, ao passo que desestimula o consumo de alimentos e preparações locais em detrimento do consumo de alimentos globalizados e ultraprocessados, especialmente entre o público jovem que busca a sensação de pertencimento a uma cultura moderna (Brasil, 2014). 
O consumo excessivo desses produtos alimentícios também tem impactos significativos na saúde, uma vez que são considerados como uma das principais causas para o desenvolvimento de doenças crônicas não transmissíveis, como diabetes, obesidade, doenças cardiovasculares e cânceres. Isso contribui para o atual cenário de maior prevalência de doenças crônicas e agrava os problemas de saúde pública (Lawrence \& Baker, 2019).

Vale destacar que os sistemas de governança dos países, incluindo o Brasil, favorecem empresas que produzem alimentos ultraprocessados. Esse apoio ocorre de diversas formas: subsídios para ingredientes por meio de commodities como milho e soja; sistemas de vigilância em saúde fragilizados ou ineficazes a respeito dos impactos na saúde humana e ambiental; acesso privilegiado da indústria aos políticos e tomadores de decisões; e estratégias de marketing agressivas (Swinburn et al., 2019).

Com base nesse último aspecto, é evidente a função da publicidade de alimentos ultraprocessados para o aumento do consumo. Os produtos alimentícios representam cerca de $20 \%$ do que é anunciado nas mídias em geral, onde as propagandas são capazes de influenciar hábitos e preferências do consumidor (Santos \& Batalha, 2010). O avanço da tecnologia, das redes sociais e a modificação nos ambientes alimentares exercem relevante impacto na influência de consumo alimentar (Hawkins, Farrow \& Thomas, 2021; Azeredo et al., 2016). Assim, o marketing representa um entrave no campo da promoção à alimentação saudável, uma vez que a ausência de regulamentação específica para a publicidade de ultraprocessados (Victora et al., 2011; Henriques, Dias \& Burlandy, 2014), presente em diversos espaços os quais os adolescentes estão inseridos, pode incentivar práticas alimentares prejudiciais à saúde humana e ambiental.

Para tanto, faz-se necessário criar normas sanitárias efetivas que regulem a comercialização e publicização dos ultraprocessados e fastfoods, bem como a formulação de Políticas Públicas no campo da alimentação e nutrição com vistas a alcançar práticas alimentares saudáveis e sustentáveis. No Brasil, ações de educação alimentar e nutricional em escolas como a Lei 13.666/18 que inseriu esse tema na Lei de Diretrizes e Bases da Educação (Brasil, 2018), o Programa Saúde na Escola (Brasil, 2007b), e o Programa Nacional de Alimentação Escolar (Brasil, 2009) devem ser espaços de promoção da alimentação saudável e sustentável com adolescentes.

\section{Considerações Finais}

O presente estudo apontou a relação direta entre o maior impacto ambiental da alimentação de adolescentes, as regiões de moradia mais urbanizadas e a maior frequência em restaurantes fast food. Avaliar essas associações é necessário para compressão das questões de saúde pública e nutrição na perspectiva da sustentabilidade para assim, serem desenvolvidas estratégias para redução da pegada hídrica e de impacto ambiental da alimentação.

Esta pesquisa possui elevada validade interna e externa por utilizar dados representativos da população de adolescentes do Brasil. Os resultados são relevantes ao produzir informações em um campo das pesquisas com poucas publicaçães sobre a investigação das pegadas ambientais da alimentação de adolescentes, mesmo não sendo uma avaliação direta do consumo alimentar, e sim uma estimativa com base em marcadores de consumo alimentar. Essa escassez de trabalhos limitou a comparação dos resultados com outras realidades. Estudos futuros devem considerar a limitação metodologia da estimativa das PH neste estudo que não pôde ser feita com base no consumo alimentar total pois a PeNSE não utiliza instrumentos de coleta como recordatórios, registros ou diários alimentares, e sim um questionário de propensão alimentar semanal.

Por fim, destaca-se que a identificação sobre como os ambientes alimentares são capazes de influenciar o consumo é necessária ao processo de vigilância alimentar e nutricional. Quando realizada com adolescentes, essas investigações geraram informações qualificadas para o desenvolvimento de ações de Saúde Coletiva e de Políticas Públicas destinadas à promoção da saúde humana e ambiental a partir de um grupo populacional com potencial para o desenvolvimento sustentável. 


\section{Referências}

Aleksandrowicz, L., Green, R., Joy, E. J. M., Smith, P., \& Haines, A. (2016). The impacts of dietary change on greenhouse gas emissions, land use, water use, and health: A systematic review. PLoS One, 11(11), 1-16. https://doi.org/10.1371/journal.pone.0165797

Andrade, G. C., Gombi-Vaca, M. F., Louzada, M. L. C., Azeredo, C. M., \& Levy, R. B. (2020). The consumption of ultra-processed foods according to eating out occasions. Public health nutrition, 23(6), 1041-1048. https://doi.org/10.1017/S1368980019002623

Azeredo, C. M., Rezende, L. F. M., Canella, D. S., Claro, R. M., Peres, M. F. T., Luiz, O. C., França-Junior, I., Kinra, S., Hawkesworth, S., \& Levy, R. B. (2016). Food environments in schools and in the immediate vicinity are associated with unhealthy food consumption among Brazilian adolescents. Preventive Medicine, 88, 73-79. https://doi.org/10.1016/j.ypmed.2016.03.026

Bezerra, I. N., Moreira, T. M. V., Cavalcante, J. B., Souza, A. M., \& Sichieri, R. (2017). Food consumed outside the home in Brazil according to places of purchase. Revista de Saúde Pública, v. 51. https://doi.org/10.1590/S1518-8787.2017051006750.

Brasil. Ministério da Saúde (MS). (2007a). Sistemas de Informações Geográficas e Análise Espacial na Saúde Pública. Brasília: MS. https://ares.unasus.gov.br/acervo/html/ARES/1198/1/livro_2.pdf

Brasil. (2007b). Decreto $\mathrm{n}^{\mathrm{o}}$ 6.286, de 5 de dezembro de 2007. Brasília: Diário Oficial da União. http://www.planalto.gov.br/ccivil_03/_ato20072010/2007/decreto/d6286.htm

Brasil. (2009). Lei $\mathrm{n}^{\mathrm{o}}$ 11.947, de 16 de junho de 2009. Brasília: Diário Oficial União. http://www.planalto.gov.br/ccivil_03/_ato20072010/2009/lei/111947.htm

Brasil. Ministério da Saúde. Conselho Nacional de Saúde. (2016). Resolução no 510 , de 07 de abril de 2016. http://conselho.saude.gov.br/resolucoes/2016/Reso510.pdf

Brasil. (2014). Guia alimentar para a população brasileira. Brasília: Ministério da Saúde, 2014. https://bvsms.saude.gov.br/bvs/publicacoes/guia_alimentar_populacao_brasileira_2ed.pdf

Brasil. (2018). Lei $\mathrm{n}^{\mathrm{o}}$ 13.666, de 16 de maio de 2018. Brasília: Diário Oficial União. http://www.planalto.gov.br/ccivil_03/_ato20152018/2018/lei/L13666.htm

Campbell, B. M., Beare, D. J., Bennett, E. M., Hall-Spencer, J. M., Ingram, J. S. I., Jaramillo, F., Ortiz, R., Ramankutty, N., Sayer, J. A., Shindell, D. et al. (2017). Agriculture production as a major driver of the Earth system exceeding planetary boundaries. Ecology and Society, 22(4), 8. https://doi.org/10.5751/ES-09595-220408

Costa, C. S., Flores, T. R., Wendt, A., Neves, R. G., Assunção, M. C. F., \& Santos, I. S. (2018). Comportamento sedentário e consumo de alimentos ultraprocessados entre adolescentes brasileiros: Pesquisa Nacional de Saúde do Escolar (PeNSE), 2015. Cadernos de Saúde Pública, $34(3)$, e00021017. https://doi.org/10.1590/0102-311X00021017

Garzillo, J. M. F., Machado, P. P., Louzada, M. L. C., Levy, R. B., \& Monteiro, C. A. (2019). Pegadas dos alimentos e das preparações culinárias consumidos no Brasil. São Paulo: Faculdade de Saúde Pública da USP. https://doi.org/10.11606/9788588848368

Gerten, D., Rockström, J., Heinke, J., Steffen, W., Richardson, K., \& Cornell, S. (2015). SUSTAINABILITY. Response to Comment on "Planetary boundaries: Guiding human development on a changing planet". Science, 348(6240), 1217. https://doi.org/10.1126/science.aab0031

Grosso, G., Fresán, U., Bes-rastrollo, M., Marventano, S., \& Galvano, F. (2020). Environmental impact of dietary choices: Role of the mediterranean and other dietary patterns in an Italian cohort. Int. J. Environ. Res. Public Health, 17, 1-13. https://doi.org/10.3390/ijerph17051468

Hallström, E., Carlsson-Kanyama, A., \& Börjesson, P. (2015). Environmental impact of dietary change: A systematic review. J. Clean. Prod., 91, 1-11. https://doi.org/10.1016/j.jclepro.2014.12.008

Han, A., Chai, L., \& Liao, X. (2020). Demographic Scenarios of Future Environmental Footprints of Healthy Diets in China. Foods, 9(8), 1021. https://doi.org/10.3390/foods9081021

Harris, F., Green, R. F., Joy, E. J. M., Kayatz, B., Haines, A., \& Dangour, A. D. (2017). The water use of Indian diets and socio-demographic factors related to dietary blue water footprint. Science of The Total Environment. 587, 128-136. https://doi.org/10.1016/j.scitotenv.2017.02.085

Hatjiathanassiadou, M., Souza, S. R. G., Nogueira, J. P., Oliveira, L. M., Strasburg, V. J., Rolim, P. M., \& Seabra, L. M. J. (2019). Environmental Impacts of University Restaurant Menus: A Case Study in Brazil. Sustainability, 11(19), 5157. https://doi.org/10.3390/su11195157

Henriques, P., Dias, P. C., \& Burlandy, L. (2014). A regulamentação da propaganda de alimentos no Brasil: convergências e conflitos de interesses. Cad Saúde Pública, 30(6), 1219-28. https://doi.org/10.1590/0102-311X00183912

Instituto Brasileiro de Geografia e Estatística (IBGE). (2010). Pesquisa de Orçamentos Familiares 2008-2009: Aquisição alimentar domiciliar per capta. Rio de Janeiro: IBGE. https://biblioteca.ibge.gov.br/index.php/biblioteca-catalogo?view=detalhes\&id=247307

Instituto Brasileiro de Geografia e Estatística (IBGE). (2016). Pesquisa Nacional de Saúde do Escolar: 2015. Rio de Janeiro: IBGE. https://biblioteca.ibge.gov.br/visualizacao/livros/liv97870.pdf

Instituto Brasileiro de Geografia e Estatística (IBGE). (2019). Pesquisa de Orçamentos Familiares: 2017-2018 - Primeiros Resultados. Rio de Janeiro: IBGE. https://biblioteca.ibge.gov.br/visualizacao/livros/liv101670.pdf

Instituto Brasileiro de Geografia e Estatística (IBGE). (2020). Pesquisa de orçamentos familiares 2017-2018: análise do consumo alimentar pessoal no Brasil. Rio de Janeiro: IBGE. https://biblioteca.ibge.gov.br/visualizacao/livros/liv101742.pdf 
Instituto Brasileiro de Geografia e Estatística (IBGE). (2021a). Séries históricas estatísticas. Taxa de urbanização: População e demografia: indicadores demográficos: 1940-2010. Disponível: https://seriesestatisticas.ibge.gov.br/series.aspx?vcodigo=POP122

Instituto Brasileiro de Geografia e Estatística (IBGE). (2021b). IBGE divulga o rendimento domiciliar per capita 2020. Disponível: https://ftp.ibge.gov.br/Trabalho_e_Rendimento/Pesquisa_Nacional_por_Amostra_de_Domicilios_continua/Renda_domiciliar_per_capita/Renda_domiciliar_p er_capita_2020.pdf

Jatobá, S.U.S. (2011). Urbanização, meio ambiente e vulnerabilidade social. Boletim Regional, Urbano e Ambiental (IPEA), 1, 141-148. http://repositorio.ipea.gov.br/bitstream/11058/5567/1/BRU_n05_urbanizacao.pdf

Lawrence, M. A., \& Baker, P. I. (2019). Ultra-processed food and adverse health outcomes. BMJ, 365, 12289. https://doi.org/10.1136/bmj.12289

Lily Hawkins, L., Farrow, C., \& Thomas, J. M. (2021). Does exposure to socially endorsed food images on social media influence food intake? Appetite, 165. https://doi.org/10.1016/j.appet.2021.105424

Monteiro, C.A., Cannon, G., Lawrence, M., Costa Louzada, M. L. \& Pereira Machado, P. (2019). Ultra-processed foods, diet quality, and health using the NOVA classification system. Rome: FAO. http://www.fao.org/3/ca5644en/ca5644en.pdf

Naja, F., Hwalla, N., Zouhbi, A. E., Abbas, N., Chamieh, M. C., Nasreddine, L. \& Jomaa, L. (2020). Changes in Environmental Footprints Associated with Dietary Intake of Lebanese Adolescents between the Years 1997 and 2009. Sustainability. 12, 4519. https://doi.org/10.1016/j.scitotenv.2017.02.085

Organización Panamericana de la Salud (OPAS). (2015). Alimentos y bebidas ultraprocesados en América Latina: tendencias, efecto sobre la obesidad e implicaciones para las políticas públicas. Washington: OPAS. https://iris.paho.org/bitstream/handle/10665.2/7698/9789275318645_esp.pdf

Santos, S. L., \& Batalha, M. O. (2010). Propaganda de alimentos na televisão: uma ameaça à saúde do consumidor? Revista de Administração, 45(4), 373-382. https://doi.org/10.1016/S0080-2107(16)30468-X

Seabra, L. M. J, Hatjiathanassiadou, M, Jorge, T. P, \& Rolim, P. M. (2021). Alimentação escolar sustentável. In: Andrade, M.E.C, \& Vale, D (org.). Caminhos para a alimentação saudável e sustentável na escola. (No prelo). Natal: Editora IFRN.

Seferidi, P., Scrinis, G., Huybrechts, I., Woods, J., Vineis, P., \& Millett, C. (2020). The neglected environmental impacts of ultra-processed foods. The Lancet Planetary Health, 4(10), e437-e438. https://doi.org/10.1016/S2542-5196(20)30177-7

Sheehan, P., Sweeny, K., Rasmussen, B., Wils, A., Friedman, H. S., Mahon, J., Patton, G. C., Sawyer, S. M., Howard, E., Symons, J., et al. (2017). Building the foundations for sustainable development: a case for global investment in the capabilities of adolescents. Lancet, 390, 1792-1806. https://doi.org/10.1016/S0140-6736(17)30872-3

Swinburn, B. A., Kraak, V. I., Allender, S., Atkins, V. J., Baker, P. I., Bogard, J. R., et al. (2019). The Lancet Commissions. The Global Syndemic of Obesity, Undernutrition, and Climate Change: The Lancet Commission report. Lancet, 393(10173), 791-846. https://doi.org/10.1016/S0140-6736(18)32822-8

Tilman, D., \& Clark, M. (2014). Global diets link environmental sustainability and human health. Nature, 515, 518-522. https://doi.org/10.1038/nature13959

United Nations. (2015). Transforming our world: the 2030 agenda for sustainable development. 2015, A/RES/70/1. https://sustainabledevelopment.un.org/post2015/transformingourworld/publication

Vale, D (org.). (2020b). Educação alimentar e nutricional de adolescentes: complexidade, resiliência e autonomia. Natal, RN: Editora IFRN, 2020. https://memoria.ifrn.edu.br/handle/1044/2089

Vale, D. (2020a). Alimentação e nutrição de adolescentes no Brasil: notas epidemiológicas. Mossoró: EDUERN. https://drive.google.com/file/d/1fqGIuaMFXJK5HOiHP5-JAW0mc4cJQc4Z/view

Vale, D., Andrade, M. E. C., Dantas, N. M., Lyra, C. O., Seabra, L. M. J., \& Roncalli, A. G. (2021). Vigilância alimentar e nutricional de adolescentes brasileiros: possibilidades com dados da PeNSE. Research, Society and Development, 10(11), e465101119818. https://doi.org/10.33448/rsd-v10i11.19818

Vale, D., Morais, C. M. M., Pedrosa, L. F. C., Ferreira, M. A. F., Oliveira, A. G. R. C., \& Lyra, C. O. (2019). Correlação espacial entre o excesso de peso, aquisição de alimentos ultraprocessados e o desenvolvimento humano no Brasil. Ciência \& Saúde Coletiva, 24(3), 983-996. https://doi.org/10.1590/141381232018243.35182016

Victora, C. G., Barreto, M. L., Carmo, L. M., Monteiro, C. A., Schmidt, M. I., Paim, J., et al. (2011). Health conditions and health-policy innovations in Brazil: the way forward. Lancet, 377, 2042-53. https://doi.org/10.1016/S0140-6736(11)60055-X

Von Koerber, K., Bader, N., \& Leitzmann, C. (2017). Wholesome nutrition: an example for a sustainable diet. Proceedings of the Nutrition Society, 76(1), 3441. https://doi.org/10.1017/S0029665116000616

Willett, W., Rockström, J., Loken, B., Springmann, M., Lang, T., Vermeulen, S., et al. (2019). Food in the Anthropocene: the EAT-Lancet Commission on healthy diets from sustainable food systems. The Lancet, 393(10170), 447-492. https://doi.org/10.1016/S0140-6736(18)31788-4 